\title{
Research on PID-Based Minimum Rule Base Fuzzy Controller in Active Joint Dental Automation
}

\author{
Farzin Matin, Hamid Cheraghi, Nasim Sobhani, Farzin Piltan and Maryam Rahmani \\ Intelligent System and Robotic Lab, Iranian Institute of Advance Science and \\ Technology (IRAN SSP), Shiraz/Iran \\ Email: piltan_f@iranssp.org,WWW.IRANSSP.ORG/english
}

\begin{abstract}
This paper describes the design intelligent based nonlinear controller for three dimensions joint dental robot manipulators in presence of uncertainty. Three-dimension joint dental robot manipulator has some important challenges such as nonlinearity, multi-input multi-output (MIMO), uncertainty in dynamic formulation, and coupling effect. To eliminate the effect of these challenge linear filter PID controller is introduce in this research. However, this controller has some advantages in certain condition but in uncertainty, it has problems. To improve this technique in presence of uncertainty, minimum rule base PID like fuzzy controller is introduced. This method reduces the risetime, steady state error and improves the trajectory performance in uncertain condition. Stability and robustness improve in proposed method as well reduce the number of rule base.
\end{abstract}

Keywords: fuzzy control, Multi degrees of freedom joints, PID Controller, minimum rule base, system's uncertainty

\section{Introduction}

The international organization defines the robot as "an automatically controlled, reprogrammable, multipurpose manipulator with three or more axes." The institute of robotic in The United States Of America defines the robot as "a reprogrammable, multifunctional manipulator design to move material, parts, tools, or specialized devices through various programmed motions for the performance of variety of tasks"[1]. Robot manipulator is a collection of links that connect to each other by joints; these joints can be one degree of freedom or multi degree of freedom. Figure 1 shows the main parts of a robot manipulator. For high precession trajectory planning and control, it is necessary to replace the actuator system made up of several single-DOF motors connected in series and/or parallel with a single multi-DOF actuator [1-3]. The spherical motor have potential contributions to a wide range of applications such as coordinate measuring, object tracking, material handling, automated assembling, welding, and laser cutting [4]. All these applications require high precision motion and fast dynamic response, which the spherical motor is capable of delivering [5-6]. The spherical motor exhibits coupled, nonlinear and very complex dynamics. The design and implementation of feedback controllers for the motor are complicated. The controller design is further complicated by the orientation-varying torque generated by the spherical motor [7]. One of the significant challenges in control algorithms is a linear behavior controller design for nonlinear systems. 


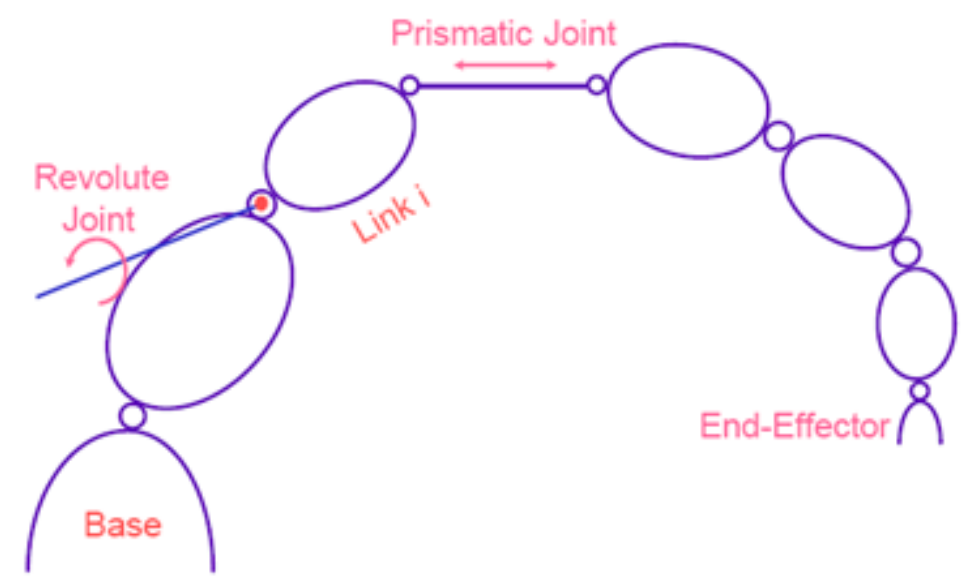

Figure 1. Main Parts of Robot Manipulator

In modern usage, the word of control has many meanings, this word is usually taken to mean regulate, direct or command. The word feedback plays a vital role in the advance engineering and science. The conceptual frame work in Feed-back theory has developed only since world war II. In the twentieth century, there was a rapid growth in the application of feedback controllers in process industries. According to Ogata, to do the first significant work in three-term or PID controllers which Nicholas Minorsky worked on it by automatic controllers in 1922. In 1934, Stefen Black was invention of the feedback amplifiers to develop the negative feedback amplifier[1-3]. Negative feedback invited communications engineer Harold Black in 1928 and it occurs when the output is subtracted from the input. Automatic control has played an important role in advance science and engineering and its extreme importance in many industrial applications, i.e., aerospace, mechanical engineering and robotic systems. The first significant work in automatic control was James Watt's centrifugal governor for the speed control in motor engine in eighteenth century[4-7].

There are several methods to control of multi degrees of freedom medical joints, which all of them follow two common goals, namely, hardware/software implementation and acceptable performance. In linear control theory, the transfer function is linear functions, which, divided into the following algorithms:

- Proportional algorithm

- Integral algorithm

- Derivative algorithm

- Proportional-Integral algorithm

- Proportional-Derivative algorithm

- Proportional-Integral-Derivative algorithm.

Control action in PID controllers can be expressed with simple model-free techniques. Given the dominance of conventional PID control in industrial control, it is significant both in theory and in practice if a controller can be found that is capable of outperforming the PID controller with comparable ease of use. Some of PID controllers are quite close to this dream. The majority of applications during the past two decades belong to the class of PID controller in industries. These controllers can be further classified into three types: the direct action (DA) type, the gain scheduling (GS) type and a combination of DA and GS types. The majority of PIDC applications belong to the DA type; here the PID controller is placed within the feedback control loop, and computes the PID coefficients through trial and error. In GS type controllers, supervisory technique is used 
to compute the individual PID gains. From the recent years, the majority of the research work on PID controllers focuses on the two-input PI or PD type controller. However, PID controller design is still a complex task due to the involvement of a large number of parameters in defining the coefficients and the rates of $K_{p}, K_{v}$ and $K_{i}$ with each others. By expressing the coefficients in different forms, each PID structure is distinctly identified. The simple analytical procedure has developed to deduce the closed form solution for a three-input controller. This solution is used to identify the PID action of each structure type in the dissociated form [1-6]. Proportional type control is used to responds immediately to difference of control input variables by immediately changing its influences variables, but this type of control is unable to eliminate the control input difference. PD controller is widely used in control process where the results are sensitive to exceeded of set point. This controller, like Proportional controller, has permanent variation in presence of self-limitation control. The Derivative component in this type of methodology is used to cancel outs the change process variables change in presence of quick change in controllers input. Integral term category, integrate the input signal deviation over a period of time. This part of controller is used to system stability after a long period of time. In contrast of Proportional type of controller, this type of controller used to eliminate the deviation. According to integral type of controller, it takes relatively long time [8-13]. The proportional type controller used to immediately response to the input variations. The proportional-integral (PI) controller has the advantages of both proportional and integral controller; it is rapid response to the input deviation as well as the exact control at the desired input. The combination of proportional (P) component, integral (I) component with a derivative (D) controller offered advantages in each case. This type of controller has rapid response to the input deviation, the exact control at the desired input as well as fast response to the disturbances. The PID controller takes the error between the desired joint variables and the actual joint variables. A proportional-derivative integral control system can easily be implemented. This method does not provide sufficient control for systems with timevarying parameters or highly nonlinear systems. An Important question which comes to mind is that why this proposed methodology should be used when lots of control techniques are accessible? Answering to this question is the main objective in this part. The problem of nonlinearity can be reduced in linear control technique, with the following two methods [14-15]:

- Limiting the performance of the system

- System linearization

To avoid of above challenges fuzzy logic methodology is used beside the PID controller. In recent years, artificial intelligence theory has been used with linear or nonlinear controller. Neural network, fuzzy logic and neuro-fuzzy are synergically combined with classical controller and improve the performance of nonlinear, time variant and uncertain plant (e.g., robot manipulator). Fuzzy logic controller (FLC) is one of the most important applications of fuzzy logic theory. This method is free of some model techniques as in model-based controllers. The main reasons to use fuzzy logic methodology are able to give approximate recommended solution for uncertain and also certain complicated systems to easy understanding and flexible. Fuzzy logic provides a method to design a model-free controller for nonlinear plant with a set of IF-THEN rules [16-22]. Fuzzy logic controls are used in many applications and they are used to modify linear and nonlinear controllers. Pure fuzzy logic controllers have problems in pre sense of uncertainty condition (robust) and pre-define the inputs/outputs gain updating factors. In fuzzy filter PID controller, fuzzy logic controller is used as an estimator for system uncertainties. To design fuzzy logic controller, PID like fuzzy logic controller is evaluated. PID like fuzzy logic controller has three inputs, Proportional (P), Derivative 
(D), and Integrator (I), which it is caused to increase the number of rule base. This challenge caused to increase the number of calculation. To solve the number of rule base in fuzzy filter PID controller parallel strategy is evaluated based on a parallel structure of a PD-like fuzzy controller and PI-like fuzzy controller. In the next step, the challenge of design PI and PD fuzzy rule tables are supposed to be solved. Design two types rule tables are very difficult therefore PD type rule table is used and PI like fuzzy logic controller is extract from PD like fuzzy logic controller. Therefore in this research PID like fuzzy controller is replaced by PD-like fuzzy controller with the integral term in output. This method is caused to design only PD type rule table for PD like fuzzy controller and PI like fuzzy controller.

This paper is organized as follows: section two, is served as an introduction to the dynamic of three degrees of freedom spherical motor, conventional Proportional, conventional integral, conventional derivative controller, conventional PD controller, conventional PI controller, and foundation of fuzzy logic algorithm. Part three, introduces and describes the methodology algorithm. Section four presents the simulation results and discussion of this algorithm and the final section describe the conclusion.

\section{Theory}

Dynamic and Kinematics Formulation of Spherical Motor: Dynamic modeling of spherical motors is used to describe the behavior of spherical motor such as linear or nonlinear dynamic behavior, design of model based controller such as pure sliding mode controller which design this controller is based on nonlinear dynamic equations, and for simulation. The dynamic modeling describes the relationship between motion, velocity, and accelerations to force/torque or current/voltage and also it can be used to describe the particular dynamic effects (e.g., inertia, coriolios, centrifugal, and the other parameters) to behavior of system. Spherical motor has nonlinear and uncertain dynamic parameters 3 degrees of freedom (DOF) motor.

The equation of a spherical motor governed by the following equation:

$$
H(q)\left[\begin{array}{c}
\ddot{\alpha} \\
\ddot{\beta} \\
\ddot{\gamma}
\end{array}\right]+\mathbf{B}(\boldsymbol{q})\left[\begin{array}{c}
\dot{\alpha} \dot{\beta} \\
\dot{\alpha} \dot{\gamma} \\
\dot{\beta} \dot{\gamma}
\end{array}\right]+\boldsymbol{C}(\boldsymbol{q})\left[\begin{array}{c}
\dot{\alpha}^{2} \\
\dot{\beta}^{2} \\
\dot{\gamma}^{2}
\end{array}\right]=\left[\begin{array}{c}
\tau_{x} \\
\tau_{y} \\
\tau_{z}
\end{array}\right]
$$

Where $\tau$ is actuation torque, $\mathrm{H}(\mathrm{q})$ is a symmetric and positive define inertia matrix, $\mathrm{B}(\mathrm{q})$ is the matrix of coriolios torques, $\mathrm{C}(\mathrm{q})$ is the matrix of centrifugal torques.

This is a decoupled system with simple second order linear differential dynamics. In other words, the component $\ddot{q}$ influences, with a double integrator relationship, only the variable $q_{i}$, independently of the motion of the other parts. Therefore, the angular acceleration is found as to be:

$$
\ddot{\boldsymbol{q}}=\boldsymbol{H}^{-1}(\boldsymbol{q}) \cdot\{\tau-\{\boldsymbol{B}+\boldsymbol{C}\}\}
$$

This technique is very attractive from a control point of view.

Study of spherical motor is classified into two main groups: kinematics and dynamics. Calculate the relationship between rigid bodies and final part without any forces is called Kinematics. Study of this part is pivotal to design with an acceptable performance controller, and in real situations and practical applications. As expected the study of kinematics is divided into two main parts: forward and inverse kinematics. Forward kinematics has been used to find the position and orientation of task frame when angles 
of joints are known. Inverse kinematics has been used to find possible joints variable (angles) when all position and orientation of task frame be active.

The main target in forward kinematics is calculating the following function:

$$
\Psi(X, q)=0
$$

Where $\Psi(.) \in R^{n}$ is a nonlinear vector function, $X=\left[X_{1}, X_{2}, \ldots \ldots, X_{l}\right]^{T}$ is the vector of task space variables which generally task frame has three task space variables, three orientation, $q=\left[q_{1}, q_{2}, \ldots, q_{n}\right]^{T}$ is a vector of angles or displacement, and finally $n$ is the number of actuated joints. The Denavit-Hartenberg (D-H) convention is a method of drawing spherical motor free body diagrams. Denvit-Hartenberg (D-H) convention study is necessary to calculate forward kinematics in this motor.

A systematic Forward Kinematics solution is the main target of this part. The first step to compute Forward Kinematics (F.K) is finding the standard D-H parameters. The following steps show the systematic derivation of the standard D-H parameters.

1. Locate the spherical motor

2. Label joints

3. Determine joint rotation $(\theta)$

4. Setup base coordinate frames.

5. Setup joints coordinate frames.

6. Determine $\alpha_{i}$, that $\alpha_{i}$, link twist, is the angle between $Z_{i}$ and $Z_{i+1}$ about an $X_{i}$.

7. Determine $d_{i}$ and $a_{i}$, that $a_{i}$, link length, is the distance between $Z_{i}$ and $Z_{i+1}$ along $X_{i} . d_{i}$, offset, is the distance between $X_{i-1}$ and $X_{i}$ along $Z_{i}$ axis.

8. Fill up the D-H parameters table. The second step to compute Forward kinematics is finding the rotation matrix $\left(R_{n}^{0}\right)$. The rotation matrix from $\left\{F_{i}\right\}$ to $\left\{F_{i-1}\right\}$ is given by the following equation;

$$
R_{i}^{i-1}=U_{i\left(\theta_{i}\right)} V_{i\left(\alpha_{i}\right)}
$$

Where $U_{i\left(\theta_{i}\right)}$ is given by the following equation;

$$
U_{i\left(\theta_{i}\right)}=\left[\begin{array}{ccc}
\cos \left(\theta_{i}\right) & -\sin \left(\theta_{i}\right) & 0 \\
\sin \left(\theta_{i}\right) & \cos \left(\theta_{i}\right) & 0 \\
0 & 0 & 1
\end{array}\right]
$$

and $V_{i\left(\alpha_{i}\right)}$ is given by the following equation;

$$
V_{i\left(\theta_{i}\right)}=\left[\begin{array}{ccc}
1 & 0 & 0 \\
0 & \cos \left(\alpha_{i}\right) & -\sin \left(\alpha_{i}\right) \\
0 & \sin \left(\alpha_{i}\right) & \cos \left(\alpha_{i}\right)
\end{array}\right]
$$

So $\left(R_{n}^{0}\right)$ is given by

$$
R_{n}^{0}=\left(U_{1} V_{1}\right)\left(U_{2} V_{2}\right) \ldots \ldots\left(U_{n} V_{n}\right)
$$

The final step to compute the forward kinematics is calculate the transformation ${ }_{n}^{0} T$ by the following formulation [3] 


$$
{ }_{n}^{0} T={ }_{1}^{0} T \cdot{ }_{2}^{1} T \cdot{ }_{3}^{2} T \ldots \ldots .{ }_{n}^{n-1} T=\left[\begin{array}{cc}
R_{n}^{0} & 0 \\
0 & 1
\end{array}\right]
$$

Linear Control Algorithm: Linear control theory is used in linear and nonlinear systems. This type of theory is used in industries, because design of this type of controller is simple than nonlinear controller. However this type of controller used in many applications but it cannot guarantee performance in complex systems. Simple linear controllers are including proportional algorithm, Proportional-Derivative algorithm, Integral algorithm, Proportional-Integral algorithm and Proportional-Integral-Derivative algorithm.

Proportional Algorithm: It is used to responds immediately to difference of control input variables by immediately changing its influences variables, but this type of control is unable to eliminate the control input difference. Figure 4 shows the block diagram of proportional controller with application to nonlinear system.

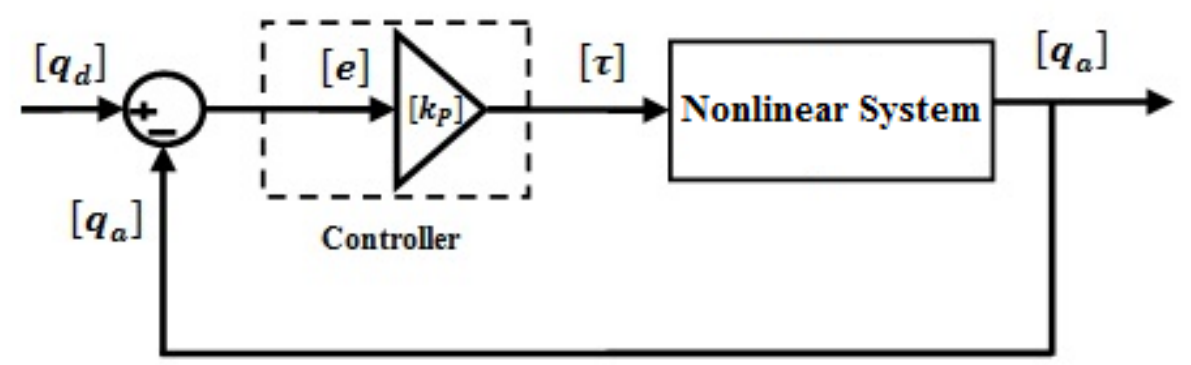

Figure 4. Block diagram of Proportional Controller

Proportional plus Derivative (PD) control: This type of linear controller is widely used in control process where the results are sensitive to exceeded of set point. This controller, like Proportional controller, has permanent variation in presence of selflimitation control. In mathematically, the formulation of Proportional-Derivative part calculated as follows;

$$
U_{P D}=K_{p} \times e+K_{v}\left(\frac{d e}{d t}\right)=K_{p} \times e+K_{v} \dot{e}
$$

The Derivative component in this type of methodology is used to cancel outs the change process variables change in presence of quick change in controllers input. Figure 5 shows the block diagram of Proportional-Derivative (PD) control of nonlinear system.

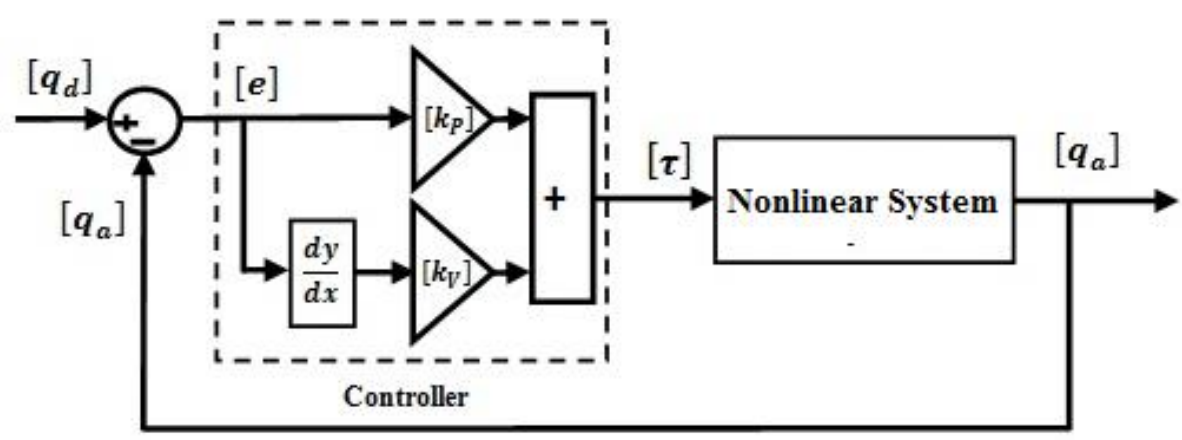

Figure 5. Block Diagram of PD Control 
Integral (I) control: This category, integrate the input signal deviation over a period of time. This part of controller is used to system stability after a long period of time. Figure 6 shows the block diagram of Integral (I) controller with application to nonlinear system. In contrast of Proportional type of controller, this type of controller used to eliminate the deviation.

In mathematically, the formulation of integral part calculated as follows;

$$
I=\frac{1}{T} \int e \cdot d t=\sum e
$$

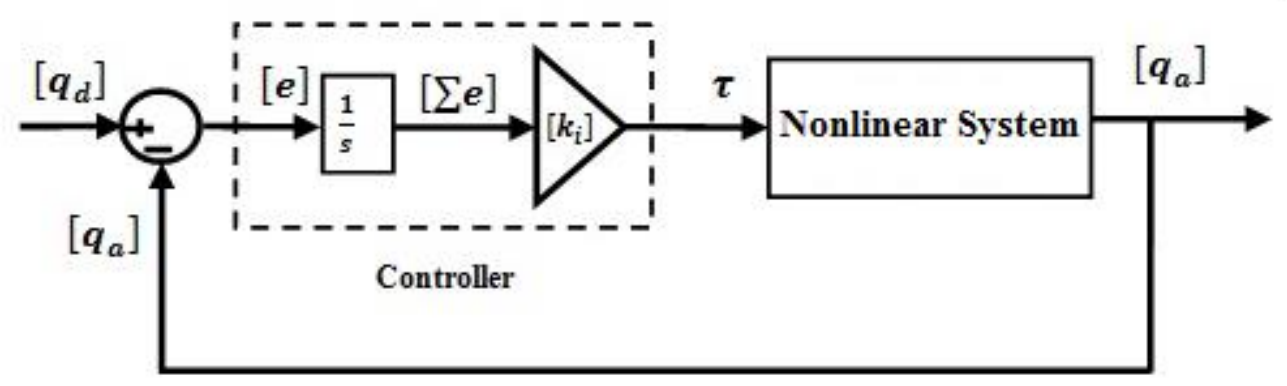

Figure 6. Block Diagram of Integral Control

Proportional plus Integral (PI) control: According to integral type of controller, it takes relatively long time. The proportional type controller used to immediately response to the input variations. The proportional-integral (PI) controller has the advantages of both proportional and integral controller; it is rapid response to the input deviation as well as the exact control at the desired input. Figure 7 shows the block diagram of PI control.

$$
U_{P I}=K_{p} \times e+K_{i}\left(\frac{1}{T} \int e . d t\right)=K_{p} \times e+K_{i} \sum e
$$

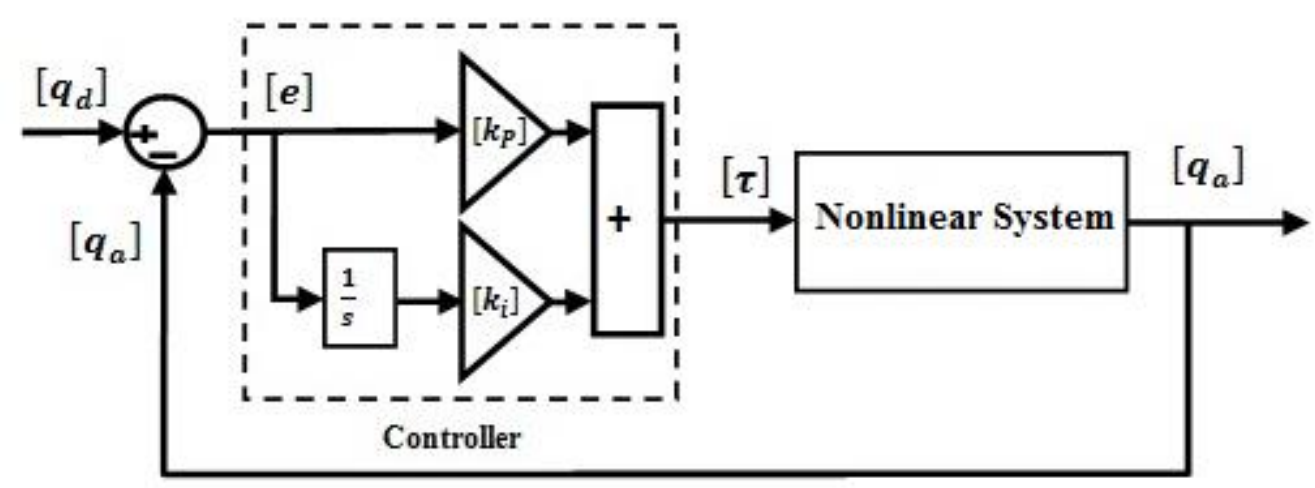

Figure 7. Block Diagram of PI Control

Fuzzy Control Algorithm: Supposed that $U$ is the universe of discourse and $x$ is the element of $U$, therefore, a crisp set can be defined as a set which consists of different elements $(x)$ will all or no membership in a set. A fuzzy set is a set that each element has a membership grade, therefore it can be written by the following definition;

$\boldsymbol{A}=\left\{\boldsymbol{x}, \mu_{A}(\boldsymbol{x}) \mid \boldsymbol{x} \in X\right\} ; \boldsymbol{A} \in \boldsymbol{U}$ 
Where an element of universe of discourse is $x, \mu_{A}$ is the membership function (MF) of fuzzy set. The membership function $\left(\mu_{A}(x)\right)$ of fuzzy set $A$ must have a value between zero and one. If the membership function $\mu_{A}(x)$ value equal to zero or one, this set change to a crisp set but if it has a value between zero and one, it is a fuzzy set. Defining membership function for fuzzy sets has divided into two main groups; namely; numerical and functional method, which in numerical method each number has different degrees of membership function and functional method used standard functions in fuzzy sets. The membership function which is often used in practical applications includes triangular form, trapezoidal form, bell-shaped form, and Gaussian form. A Trapezoidal membership function of fuzzy set is defined by the following equation

$\boldsymbol{\mu}_{\boldsymbol{F}(\boldsymbol{x})}=\left\{\begin{array}{cl}\mathbf{0} \boldsymbol{\boldsymbol { x }}, & x<a \\ \frac{\boldsymbol{b}-\boldsymbol{a}}{\boldsymbol{d}-\boldsymbol{x}}, & a \leq x<b \\ \hline \boldsymbol{d - c}, & c \leq x<d \\ \mathbf{0}, & x>d\end{array}\right.$

A Triangular membership function of fuzzy set is defined by the following equation

$\boldsymbol{\mu}_{\boldsymbol{F}(\boldsymbol{x})}=\left\{\begin{array}{cl}\mathbf{0}, \boldsymbol{x}, & x<a \\ \frac{\boldsymbol{b}-\boldsymbol{a}}{\boldsymbol{c}-\boldsymbol{x}}, & a \leq x<b \\ \hline \boldsymbol{c - \boldsymbol { b }}, & b \leq x \leq c \\ \mathbf{0}, & x>c\end{array}\right.$

A Gaussian membership function of fuzzy set is defined by

$\mu_{F(x)}=e^{\frac{-\left(x-c_{F}\right)^{2}}{W}}$

and a Bell-shaped membership function of fuzzy set is defined by

$\mu_{F(x)}=\frac{1}{1+\left(x-c_{F}\right)^{2}}$

Figure 8 shows the typical shapes of membership functions in a fuzzy set

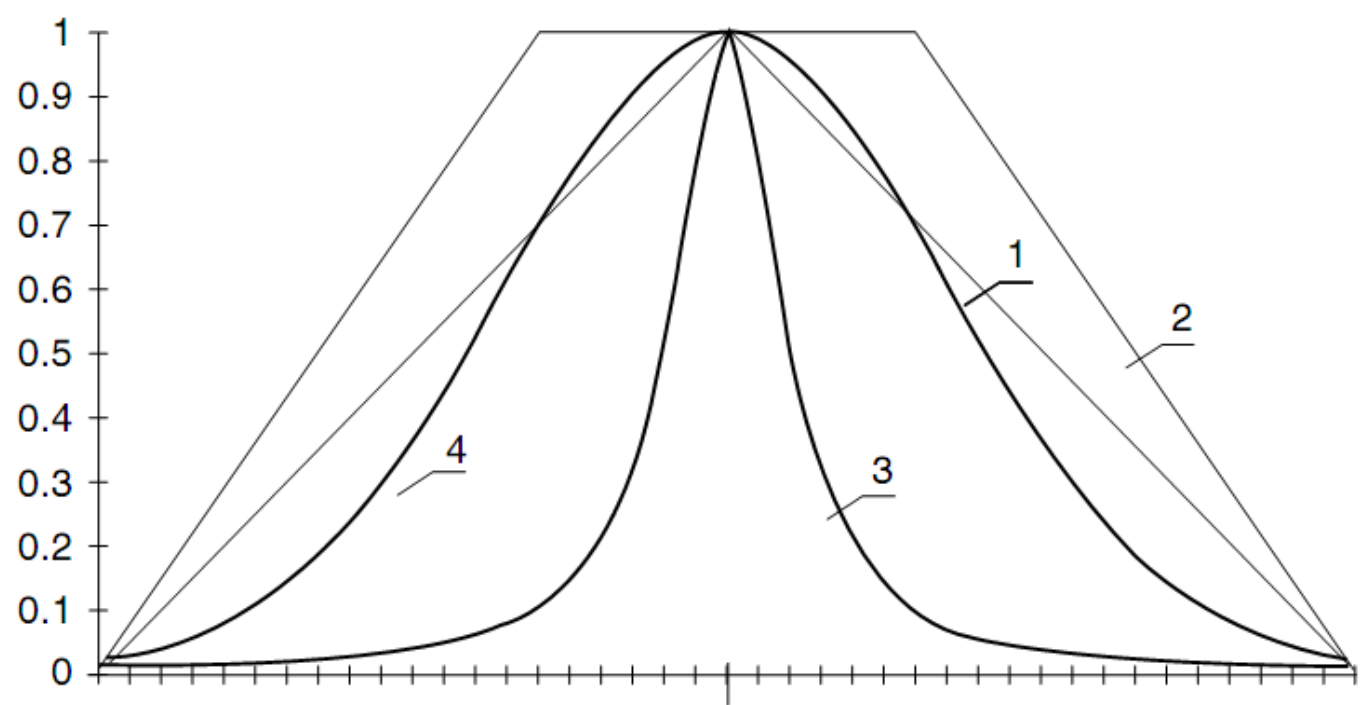

Figure 8. Membership Functions in Fuzzy set: 1-Trianglar, 2-Trapezoidal, 3Gaussian, 4-Bell-shaped 
The union of two fuzzy set $A$ and $B(S-$ norm) is a new fuzzy set which the new membership function is given by

$\boldsymbol{S}(\boldsymbol{a}, \boldsymbol{b})=\boldsymbol{\mu}_{A \cup B(u)}=\max \left\{\mu_{A(u)}, \mu_{B(u)}\right\}, \quad \forall u \in U$

The intersection of two fuzzy set $A$ and $B(T-$ norm) is a new fuzzy set which the new membership function is given by

$$
\begin{aligned}
& T(a, b)=\mu_{A \cap B(u)}=\min \left\{\mu_{A(u)}, \mu_{B(u)}\right\}=\mu_{A(u)} \cdot \mu_{B(u)} \\
& =\max \left(0, \mu_{A(u)}+\mu_{B(u)}-1\right)=\left\{\begin{array}{lr}
\mu_{A(u)}, & \text { if } \mu_{B(u)}=1 \\
\mu_{B(u)}, & \text { if } \mu_{A(u)}=1 \\
0 & , \text { if } \mu_{B(u)}, \mu_{A(u)}<1
\end{array}\right.
\end{aligned}
$$

In fuzzy set the min operation can resolve the statement $A A N D B$ and can be shown by $\min (A, B)$ operation. Using the same reason, the $A O R B$ operation can be replace by $\max$ operation in fuzzy set and at last the NOT $A$ operation can be replace by $1-A$ operation in fuzzy set. The algebraic product of two fuzzy set $A$ and $B$ is the multiplication of the membership functions which is given by the following equation

$\mu_{A \cdot B(u)}=\mu_{A(u)} \cdot \mu_{B(u)}$

The algebraic Sum of two fuzzy sets $A$ and $B$ is given by the following equation

$\mu_{A \hat{+} B(u)}=\mu_{A(u)} \cdot \mu_{B(u)}-\mu_{A(u)} \cdot \mu_{B(u)}$

Linguistic variable can open a wide area to use of fuzzy logic theory in many applications (e.g., control and system identification). In a natural artificial language all numbers replaced by words or sentences. In Figure 9 the linguistic variable is torque and the linguistic values are Low,Medium and High.

If - then Rule statements are used to formulate the condition statements in fuzzy logic. A single fuzzy If - then rule can be written by

\section{If $x$ is $A$ Then $y$ is $B$}

where $A$ and $B$ are the Linguistic values that can be defined by fuzzy set, the If - part of the part of " $x$ is $A$ " is called the antecedent part and the then - part of the part of " $y$ is $B$ " is called the Consequent or Conclusion part. The antecedent of a fuzzy if-then rule can have multiple parts, which the following rules shows the multiple antecedent rules:

if $e$ is $N B$ and $\dot{e}$ is $M L$ then $T$ is $L L$

where $e$ is error, $\dot{e}$ is change of error, $N B$ is Negative Big, $M L$ is Medium Left, $T$ is torque and $L L$ is Large Left. 


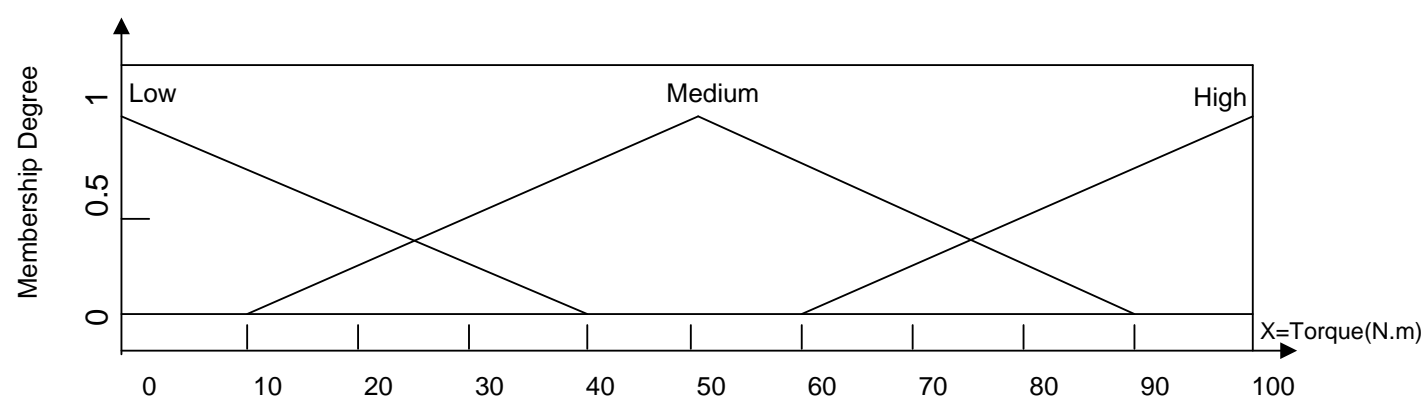

Figure 9. Linguistic Variable and Linguistic Value

If - then rules have three parts, namely, fuzzify inputs, apply fuzzy operator and apply implication method which in fuzzify inputs the fuzzy statements in the antecedent replaced by the degree of membership, apply fuzzy operator used when the antecedent has multiple parts and replaced by single number between 0 to 1 , this part is a degree of support for the fuzzy rule, and apply implication method used in consequent of fuzzy rule to replaced by the degree of membership. Figure 10 shows the main three parts in If - then rules.

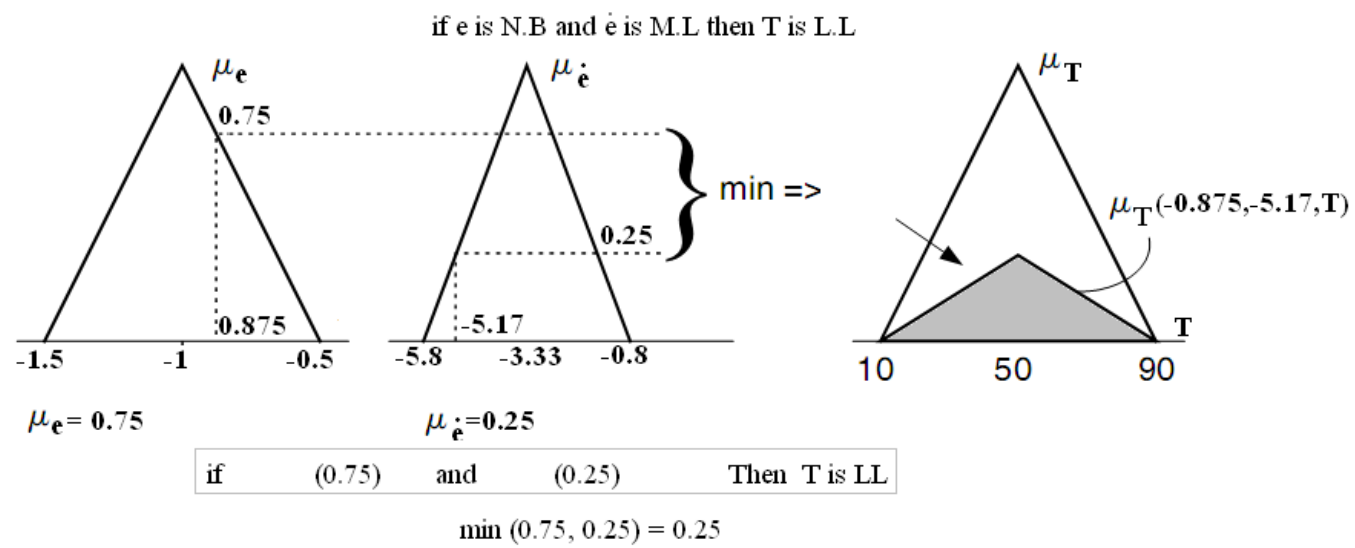

Figure 10. Main three Parts in IF-THEN Rules in Fuzzy Set

The fuzzy inference engine offers a mechanism for transferring the rule base in fuzzy set which it is divided into two most important methods, namely, Mamdani method and Sugeno method. Mamdani method is one of the common fuzzy inference systems and he designed one of the first fuzzy controllers to control of system engine. Mamdani's fuzzy inference system is divided into four major steps: fuzzification, rule evaluation, aggregation of the rule outputs and defuzzification. Michio Sugeno use a singleton as a membership function of the rule consequent part. The following definition shows the Mamdani and Sugeno fuzzy rule base
Mamdani
$F . R^{1}:$ if
$x$ is $A$ and $y$ is $B$ then
$z$ is $C$
Sugeno
F. $R^{1}$ :if $x$ is A and $y$ is $B$ then $f(x, y)$ is $C$

When $x$ and $y$ have crisp values fuzzification calculates the membership degrees for antecedent part. Rule evaluation focuses on fuzzy operation $(A N D / O R)$ in the antecedent of the fuzzy rules. The aggregation is used to calculate the output fuzzy set and several methodologies can be used in fuzzy logic controller aggregation, namely, Max-Min aggregation, Sum-Min aggregation, Max-bounded product, Max-drastic 
product, Max-bounded sum, Max-algebraic sum and Min-max. Two most common methods that used in fuzzy logic controllers are Max-min aggregation and Sum-min aggregation. Max-min aggregation defined as below

$$
\mu_{U}\left(x_{k}, y_{k}, U\right)=\mu_{\cup i=1}^{r} F R^{i}\left(x_{k}, y_{k}, U\right)=\max \left\{\min _{i=1}^{r}\left[\mu_{R p q}\left(x_{k}, y_{k}\right), \mu_{p_{m}}(U)\right]\right\}
$$

The Sum-min aggregation defined as below

$\mu_{U}\left(x_{k}, y_{k}, U\right)=\mu_{\cup_{i=1}^{r} F R^{i}}\left(x_{k}, y_{k}, U\right)=\sum \min _{i=1}^{r}\left[\mu_{R p q}\left(x_{k}, y_{k}\right), \mu_{p_{m}}(U)\right]$

where $r$ is the number of fuzzy rules activated by $x_{k}$ and $y_{k}$ and also $\mu_{\cup_{i=1}^{r} F R^{i}}\left(x_{k}, y_{k}, U\right)$ is a fuzzy interpretation of $i-t h$ rule. Defuzzification is the last step in the fuzzy inference system which it is used to transform fuzzy set to crisp set. Consequently defuzzification's input is the aggregate output and the defuzzification's output is a crisp number. Centre of gravity method $(C O G)$ and Centre of area method $(C O A)$ are two most common defuzzification methods, which COG method used the following equation to calculate the defuzzification

$\operatorname{COG}\left(x_{k}, y_{k}\right)=\frac{\sum_{i} U_{i} \sum_{j=1}^{r} \cdot \mu_{u}\left(x_{k}, y_{k}, U_{i}\right)}{\sum_{i} \sum_{j=1}^{r} \cdot \mu_{u}\left(x_{k}, y_{k}, U_{i}\right)}$

and $C O A$ method used the following equation to calculate the defuzzification

$\operatorname{COA}\left(x_{k}, y_{k}\right)=\frac{\sum_{i} U_{i} \cdot \mu_{u}\left(x_{k}, y_{k}, U_{i}\right)}{\sum_{i} \mu_{U} \cdot\left(x_{k}, y_{k}, U_{i}\right)}$

Where $\operatorname{COG}\left(x_{k}, y_{k}\right)$ and $\operatorname{COA}\left(x_{k}, y_{k}\right)$ illustrates the crisp value of defuzzification output, $U_{i} \in U$ is discrete element of an output of the fuzzy set, $\mu_{U} .\left(x_{k}, y_{k}, U_{i}\right)$ is the fuzzy set membership function, and $r$ is the number of fuzzy rules.

Based on foundation of fuzzy logic methodology; fuzzy logic controller has played important rule to design nonlinear controller for nonlinear and uncertain systems [53]. However the application area for fuzzy control is really wide, the basic form for all command types of controllers consists of;

- Input fuzzification (binary-to-fuzzy[B/F]conversion)

- Fuzzy rule base (knowledge base)

- Inference engine

- Output defuzzification (fuzzy-to-binary[F/B]conversion).

The basic structure of a fuzzy controller is shown in Figure 11. 


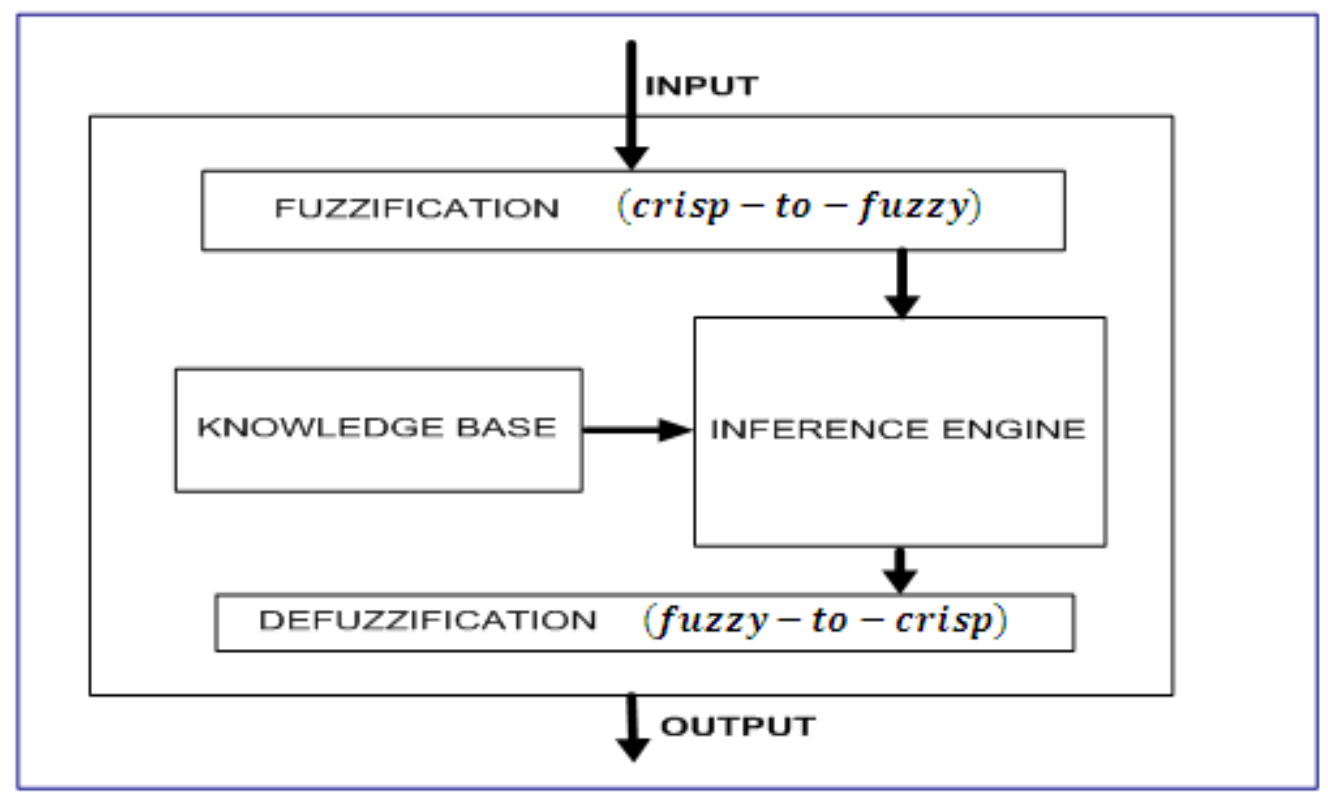

Figure 11. Structure of Fuzzy Logic Controller (FLC)

Based on Figure 11; fuzzification is used to change the crisp set into fuzzy set. Knowledge base is used to rule evaluation and determine the membership degree and if all fuzzy inputs activated by the known input values. Fuzzy inference engine is used to transferring the rule base into fuzzy set by Mamdani's or Sugeno method based on aggregation of the rules output. Deffuzification is the last part to calculate the fuzzy inference system.

\section{Methodology}

The combination of proportional (P) component, integral (I) component with a derivative (D) controller offered advantages in each case. This type of controller has rapid response to the input deviation, the exact control at the desired input as well as fast response to the disturbances. The PID controller takes the error between the desired joint variables and the actual joint variables to control. A proportional-derivative integral control system can easily be implemented. This method does not provide sufficient control for systems with time-varying parameters or highly nonlinear systems. Figure 12 shows the block diagram of PID control. The formulation of PID controller calculated as follows;

$U_{P I D}=K_{p} \times e+K_{i}\left(\frac{1}{T} \int e . d t\right)+K_{v}\left(\frac{d e}{d t}\right)=K_{p} \times e+K_{i} \sum e+K_{v} \dot{e}$ 


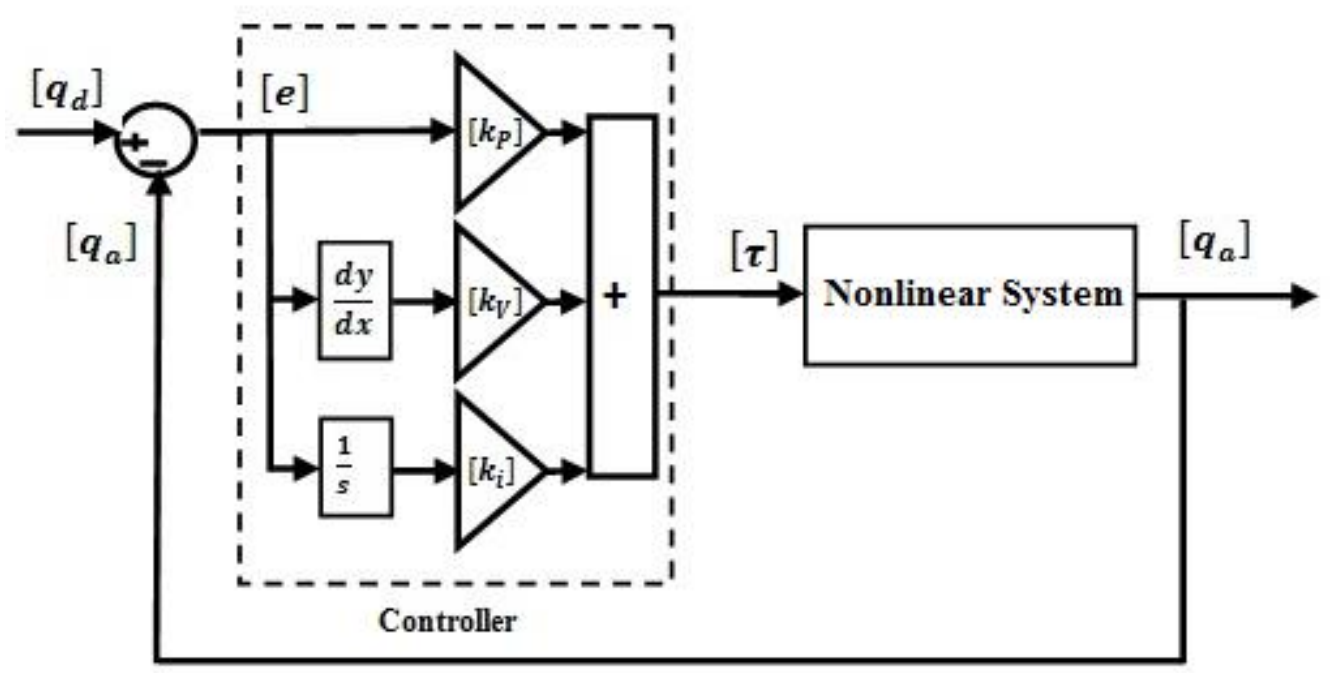

Figure 12. Block Diagram of PID Control of Multi Degrees of Freedom

Proportional-Integral-Derivative (PID) controller has rapid response to the input deviation, the exact control at the desired input as well as fast response to the disturbances. The PID controller takes the error between the desired joint variables and the actual joint variables to control the three dimension of joint. The equation of PID controller for control of 3 degrees of freedom joint is;

$\left[\begin{array}{l}\widehat{\tau_{1}} \\ \widehat{\tau_{2}} \\ \widehat{\tau_{3}}\end{array}\right]=\left[\begin{array}{l}K_{i 1} \sum e_{1}+K_{v 1} \dot{e}_{1}+K_{p 1} e_{1} \\ K_{i 2} \sum e_{2}+K_{v 2} \dot{e}_{2}+K_{p 2} e_{2} \\ K_{i 3} \sum e_{3}+K_{v 3} \dot{e}_{3}+K_{p 3} e_{3}\end{array}\right]$

Where $e=q_{d}-q_{a}, q_{d}$ is desired joint variable and $q_{a}$ is actual joint variable.

In PID controller the control law is given by the following equation;

$\tau=K_{p} e+K_{v} \dot{e}+K_{i} \sum e$

Where $\boldsymbol{e}=\boldsymbol{q}_{\boldsymbol{i}_{\boldsymbol{d}}}-\boldsymbol{q}_{\boldsymbol{i}_{\boldsymbol{a}}}$

In this theory $\boldsymbol{K}_{\boldsymbol{p}}, \boldsymbol{K}_{\boldsymbol{i}}$ and $\boldsymbol{K}_{\boldsymbol{v}}$ are positive constant. To show this controller is stable and achieves zero steady state error, the Lyapunov function is introduced;

$V=\frac{1}{2}\left[\dot{q}^{T} A(q) \dot{q}+e^{T} K_{p} e\right]=$

$\frac{1}{2} \frac{d}{d t}\left[\dot{q}^{T} A \dot{q}\right]=\dot{q} \tau$

If the conversation energy is written by the following form:

$\frac{1}{2} \frac{d}{d t}\left[\dot{q}^{T} A \dot{q}\right]=\dot{q} \tau$ 
Where $(\dot{\boldsymbol{q}} \boldsymbol{\tau})$ shows the power inputs from actuator and

$\frac{1}{2} \frac{d}{d t}\left[\dot{\boldsymbol{q}}^{\boldsymbol{T}} \boldsymbol{A} \dot{\boldsymbol{q}}\right]$ is the derivative of the robot kinematic energy.

$\dot{V}=\dot{q}^{T}\left[\tau+K_{p} e\right]$

Based on $\boldsymbol{\tau}=-\boldsymbol{K}_{\boldsymbol{p}_{\boldsymbol{i}}} \boldsymbol{e}-\boldsymbol{K}_{\boldsymbol{v}_{\boldsymbol{i}}} \dot{\boldsymbol{e}}-\boldsymbol{K}_{\boldsymbol{i}} \sum \boldsymbol{e}$, we can write:

$\dot{V}=\dot{q}^{T} K_{p} \dot{q} \leq \mathbf{0}$

If $\dot{\boldsymbol{V}}=\mathbf{0}$, we have

$\dot{q}=0 \rightarrow \ddot{q}=0 \rightarrow \ddot{q}=A^{-1} K_{p} e \rightarrow e=0$

In this state, the actual trajectories converge to the desired state. To improve the system performance as well stability based on pure PID controller we have the following challenge:

- Limiting the performance of the system according to the required velocities and accelerations.

Regarding to type of system, the rate of speed is the main objective in this research. To improve the performance of PID controller as well stability surface design is introduced. Uncertainties are very important challenges and caused to overestimation of the bounds. As this point if $S=K_{1} e+\dot{e}=0$ is chosen as desired surface, if the system's dynamic is derived to surface and used to reduce the challenge of uncertainty then the linearization and decoupling through the use of feedback, can be realized. Because, when the system dynamic is on the surface the derivative of surface $\dot{S}=K_{1} \dot{e}+\ddot{e}$ is equal to the zero that is a decoupled and linearized closed-loop system dynamics. Linearization and decoupling by the above method can be obtained in spite of the quality of the system dynamic model. It is well known fact that if the uncertainties are very good compensate there is no need to use adaptive control which create the high computation burden.

According to the theory, the main important part to design this controller is surface, a time-varying surface $\boldsymbol{s}(\boldsymbol{x}, \boldsymbol{t})$ in the state space $\boldsymbol{R}^{\boldsymbol{n}}$ is given by the following formulation:

$s(x, t)=\left(\frac{d}{d t}+\lambda\right)^{n-1} \tilde{x}=0$

$\lambda$ is the surface slope coefficient and it is positive constant. The surface can be defined as Proportional-Derivative (PD), Proportional-Integral (PI) and the Proportional-IntegralDerivative (PID). The following formulations represented the three groups are:

$S_{P D}=\lambda e+\dot{e}$

$s(x, t)=\left(\frac{d}{d t}+\lambda\right)^{n-1}\left(\int_{0}^{t} \tilde{x} d t\right)=0$

$S_{P I}=\lambda e+\left(\frac{\lambda}{2}\right)^{2} \sum e$

$S_{P I D}=\lambda e+\dot{e}+\left(\frac{\lambda}{2}\right)^{2} \sum e$

To have the stability and minimum error, the main objective is kept the surface slope $s(x, t)$ near to the zero. Therefore, one of the common strategies is to find input $U$ outside of $s(x, t)$. 
$\frac{1}{2} \frac{d}{d t} s^{2}(x, t) \leq-\zeta|s(x, t)|$

$\zeta$ is positive constant.

If $S(0)>0 \rightarrow \dot{S}(t) \leq-\zeta$

Derivative term of $(\boldsymbol{s})$ is eliminated by limited integral from $\mathrm{t}=0$ to $\mathrm{t}=\boldsymbol{t}_{\text {reach }}$

$\int_{t=0}^{t=t_{\text {reach }}} \dot{S}(t) \leq-\int_{t=0}^{t=t_{\text {reach }}} \eta \rightarrow S\left(t_{\text {reach }}\right)-S(0) \leq-\zeta\left(t_{\text {reach }}-0\right)$

$t_{\text {reach }}$ is the time that trajectories reach to the surface. If $\boldsymbol{S}_{\boldsymbol{t}_{\text {reach }}}=\mathbf{0}$ the formulation of $\boldsymbol{t}_{\text {reach }}$ calculated by;

$0-S(0) \leq-\eta\left(t_{\text {reach }}\right) \rightarrow t_{\text {reach }} \leq \frac{S(0)}{\zeta}$

If $\boldsymbol{S}(\mathbf{0})<0$

$0-S(0) \leq-\eta\left(t_{\text {reach }}\right) \rightarrow S(0) \leq-\zeta\left(t_{\text {reach }}\right) \rightarrow t_{\text {reach }} \leq \frac{|S(0)|}{\eta}$

This formulation is guarantee time to reach the sliding surface is smaller than $\frac{|\boldsymbol{S}(\mathbf{0})|}{\zeta}$ since the trajectories are outside of $S(t)$.

if $S_{t_{\text {reach }}}=S(0) \rightarrow \operatorname{error}\left(x-x_{d}\right)=0$

$s(x, t)=\left(\frac{d}{d t}+\lambda\right) \quad \widetilde{x}=\left(\dot{\mathbf{x}}-\dot{\mathbf{x}}_{\mathrm{d}}\right)+\lambda\left(\mathbf{x}-\mathbf{x}_{\mathbf{d}}\right)$

The change of sliding surface $(\dot{S})$ is;

$\dot{\boldsymbol{S}}=\left(\ddot{\mathbf{x}}-\ddot{\mathbf{x}}_{\mathbf{d}}\right)+\lambda\left(\dot{\mathbf{x}}-\dot{\mathbf{x}}_{\mathbf{d}}\right)$

$\dot{S}=\lambda \dot{e}+\ddot{e}$

The final formulation of control design is:

$U_{\text {proposed }}=K_{p} \times e+K_{i} \sum e+K_{v} \dot{e}+s(x, t)+s(\dot{x}, t)$

PID like fuzzy logic controller has three inputs, Proportional (P), Derivative (D), and Integrator (I), if each input defined by $N$ linguistic variables to estimate the dynamic behavior, it has $N \times N \times N$ linguistic variables. Design fuzzy controller based on $N^{3}$ rule base caused to creation lots of challenges in real timing application. To reduce the number of rule base in PID like fuzzy logic controller, parallel PD and PI strategy is 
recommended. According to this algorithm to design the same PID controller the number of rule base for each link is $2 N^{2}$. According to this technique, the number of rule base is reduced with respect to have PID like fuzzy logic controller. After solve the first challenge about the number of rule base in PID like fuzzy logic controller, the second challenge is appears. To design parallel PD and PI like fuzzy controller, two types fuzzy rule table should be design. Design two types rule tables are very difficult and need to have much experience. Therefore in this research PI-like fuzzy controller is replaced by PD-like fuzzy controller with the integral term in the output. Due to this method, researcher can design PID like fuzzy logic controller based on PD rule table and $2 N^{2}$ rule base.

According to fuzzy logic methodology definition;

$\boldsymbol{U}_{\text {fuzzy }}=\left(\sum_{l=1}^{\boldsymbol{M}} \boldsymbol{\theta}^{\boldsymbol{T}} \boldsymbol{\zeta}(\boldsymbol{x})\right)_{e, \sum e, \dot{e}}$

where $\boldsymbol{\theta}^{\boldsymbol{T}}$ is gain updating factor and $\boldsymbol{\zeta}(\boldsymbol{x})$ is defined by;

$\zeta(x)=\frac{\sum_{i} \mu\left(x_{i}\right) x_{i}}{\sum_{i} \mu\left(x_{i}\right)}$

And the $\boldsymbol{\mu}\left(\boldsymbol{x}_{\boldsymbol{i}}\right)$ parameter is membership function.

Figure 13 illustrates the general structure of the PID like fuzzy logic controller, which consists of two main components. The PID like fuzzy logic controller is built using PD like fuzzy logic controller and PI like fuzzy logic controller. According to design PD like fuzzy controller and PI like fuzzy controller based on PD fuzzy rule base, design PID like fuzzy controller is introduced based on the following formulation;

$U_{P I D}=U_{P I}+U_{P D}=\left(\frac{K_{p}}{2}\right) \times e+K_{i}\left(\frac{1}{T} \int e . d t\right)+\left(\frac{K_{p}}{2}\right) \times e+K_{v} \dot{e}$
$U_{P I D l i k e}$ fuzzy $=\left[\left(\sum_{l=1}^{M} \theta^{T} \zeta(x)\right)_{e, \sum e}+\left(\sum_{l=1}^{M} \theta^{T} \zeta(x)\right)_{e, \dot{e}}\right]$

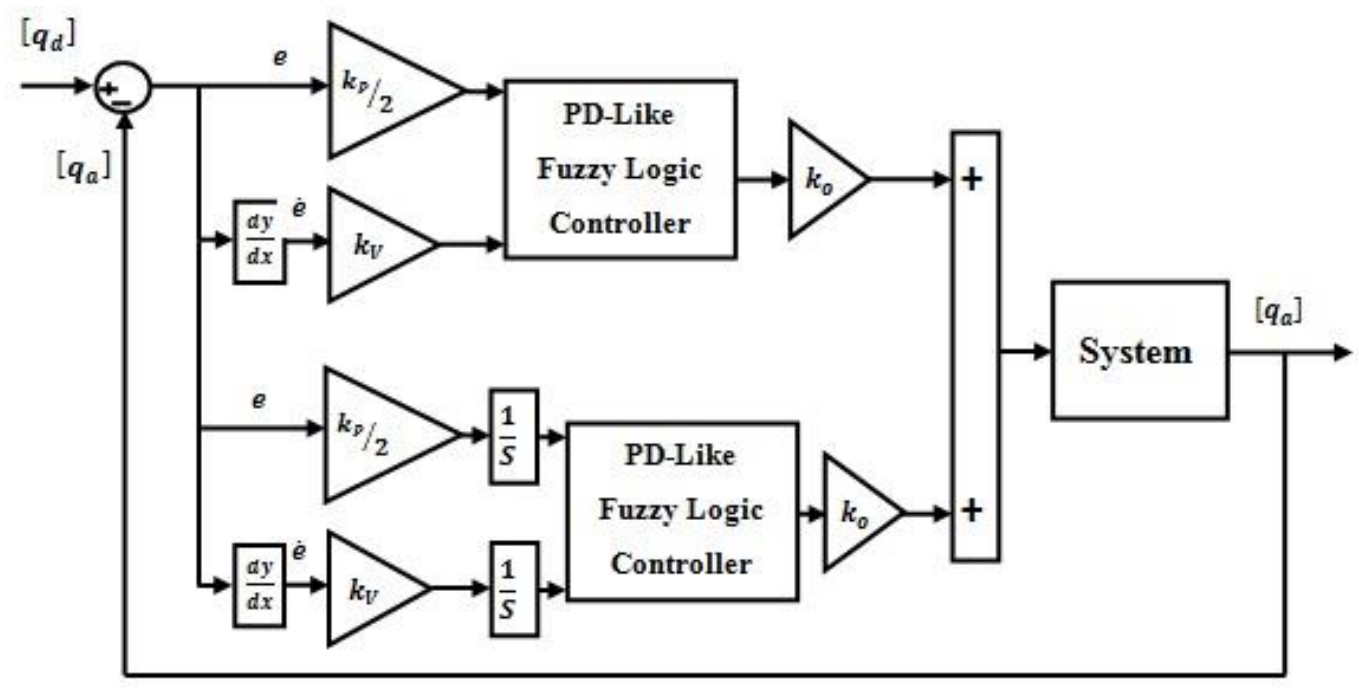

Figure 13. Design PID like Fuzzy Controller

PID like fuzzy logic controllers consists of the following parts;

- Choosing inputs

- Scaling inputs

- Input fuzzification (binary-to-fuzzy[B/F] conversion)

- Fuzzy rule base (knowledge base)

- Inference engine 
- Output defuzzification (fuzzy-to-binary[F/B] conversion)

- Scaling output

Define the inputs and control variables: In PID-like fuzzy controller, error and change of error are used to define as controllers' inputs. Therefore the antecedent part of rule base is comprised of two parts. In this part fuzzy controller's inputs are error $(e)$ and change of error $(\dot{e})$ and the fuzzy controller output is PD fuzzy output $\left(U_{P D-f u z z y}\right)$ and PI fuzzy output $\left(U_{P I-f u z z y}\right)$.

Scaling variables and Input fuzzification (binary-to-fuzzy [B/F] conversion):

Proposed PID like fuzzy logic controller has two inputs error, change of error and two different output; PD fuzzy output and PI fuzzy output. Error defined as seven linguistic variables: Negative Big (NB), Negative Medium (NM), Negative Small (NS), Zero (Z), Positive Small (PS), Positive Medium (PM), Positive Big (PB). Based on experience knowledge the range of scaling factor for error is [-0.1 to 0.1$]$ and it is quantized into eleven levels as follows: $e=\{-0.1,-0.08,-0.06,-0.04,-0.02,0,0.02,0.04,0.06,0.08,0.1\}$. The linguistic values for change of error are: Negative $(\mathrm{N})$, Zero $(\mathrm{Z})$ and Positive $(\mathrm{P})$ and the range of scaling factor for change of error is [ -1 to 1$]$ and it is quantized into eleven levels as: $\dot{e}=\{-1,-0.8,-0.6,-0.4,-0.2,0,0.2,0.4,0.6,0.8,1\}$. The linguistic variables for PID like fuzzy logic controller's output divided into two main parts, PD like fuzzy logic controller and PI like fuzzy logic controller which, linguistic variables for output PD like fuzzy logic controller and PI like fuzzy logic controller are: Negative Big (NB), Negative Medium (NM), Negative Small (NS), Zero (Z), Positive Small (PS), Positive Medium $(\mathrm{PM})$, Positive Big (PB) and the scaling factor for them are [-1.5 to 1.5].

Fuzzy rule Base: the main approach comes from an expert knowledge of system because fuzzy controller is one of the expert system to solve the control problem. According to fuzzification the error has seven linguistic variables, the change of error has three linguistic variables and the PD fuzzy output and PI like fuzzy logic controller have seven linguistic variables. Therefore PID like fuzzy controller has 42 rule-bases. The PID like fuzzy rule table shows in Table 1.

Table 1. Rule Table in PID like Fuzzy Logic Controller

\begin{tabular}{|c|c|c|c|c|c|c|c|}
\hline$\dot{\boldsymbol{e}}$ & $\boldsymbol{P B}$ & $\boldsymbol{P M}$ & $\boldsymbol{P S}$ & $\boldsymbol{Z}$ & $\boldsymbol{N S}$ & $\boldsymbol{N} \boldsymbol{M}$ & $\boldsymbol{N B}$ \\
\hline $\boldsymbol{P}$ & $\mathrm{NB}$ & $\mathrm{NB}$ & $\mathrm{NB}$ & $\mathrm{NB}$ & $\mathrm{N}$ & $\mathrm{NS}$ & $\mathrm{Z}$ \\
\hline $\boldsymbol{Z}$ & $\mathrm{NB}$ & $\mathrm{NM}$ & $\mathrm{NS}$ & $\mathrm{Z}$ & $\mathrm{PS}$ & $\mathrm{PM}$ & $\mathrm{PB}$ \\
\hline $\boldsymbol{N}$ & $\mathrm{Z}$ & PS & PM & PB & PB & PB & PB \\
\hline
\end{tabular}

Inference Engine (Fuzzy rule processing): In this research 42 rule bases Mamdani fuzzy inference engine is used as fuzzy rule processing.

Defuzzification: defuzzification is the last step to design fuzzy logic controller and it is used to transform fuzzy set to crisp set. In PID like fuzzy logic controller COG method is used for defuzzification. 


\section{Results and Discussion}

In this part, conventional PID controller and filter PID fuzzy logic controller are compared and test using MATLAB/SIMULINK. Trajectory tracking, power of disturbance rejection, time of response and error are test in this part.

Trajectory following: conventional PID controller and filter PID fuzzy controller and reference signal are test and show in Figure 14. Based on the following Figure, conventional PID controller has 5\% overshoot, rise time in this controller is 0.82 second and the steady state error in $\mathrm{t}=30$ second is 0.001 . Proposed method has $0 \%$ overshoot, it does not have rise time and the steady sate error in 30 second is about zero. In certain condition, proposed method has excellent response.

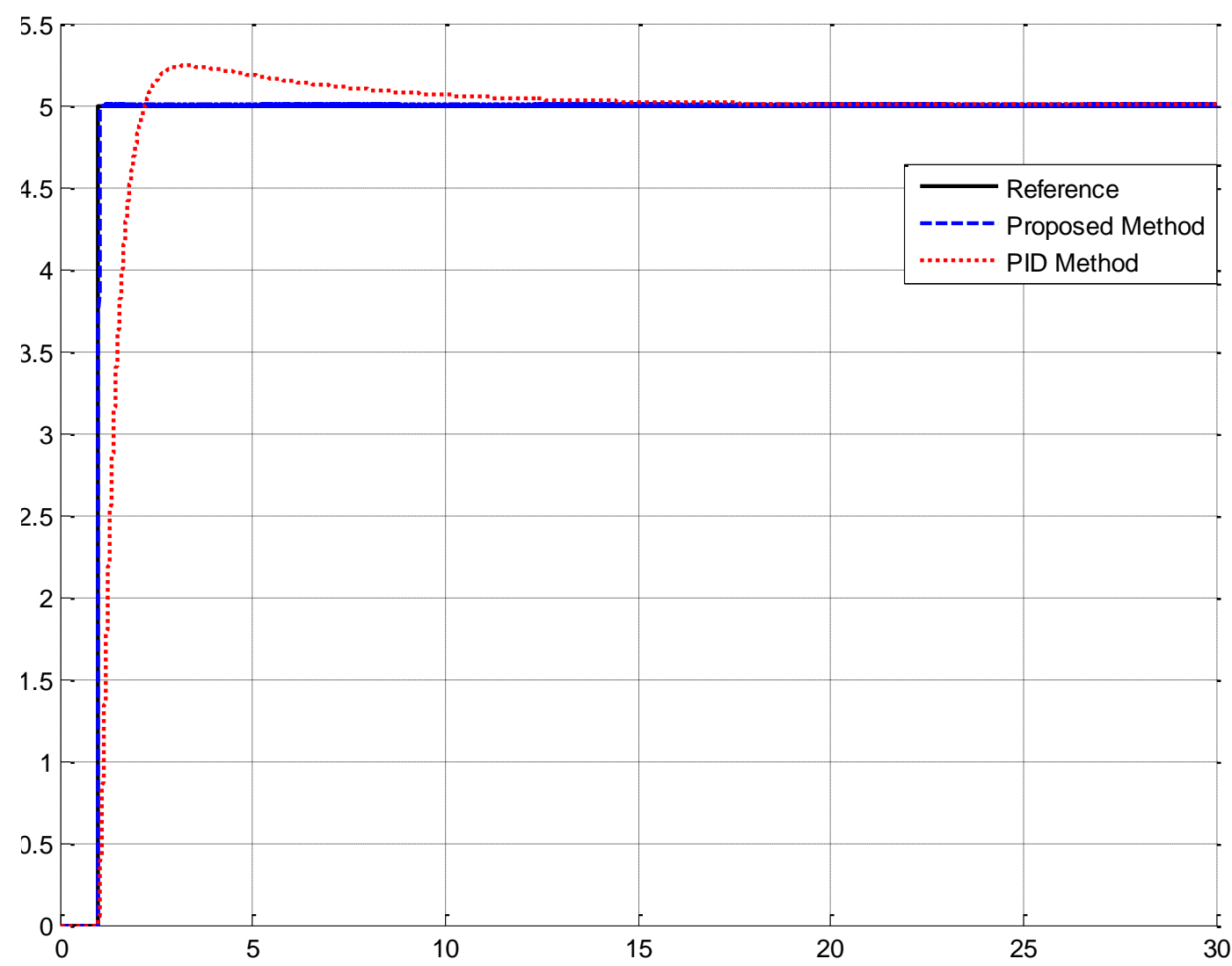

Figure 14. Trajectory following for PID Controller and Proposed Method in Certain Condition

Power of disturbance rejection: the power of disturbance rejection is very important to robust checking in this paper. To test disturbance rejection, this research test unstructured uncertainty. Regarding to Figure 15, conventional PID controller has $40 \%$ overshoot, rise time is about 1.3 second and steady state error at 30 second is about 0.001 . Based on these information's, in uncertain condition overshoot and rise time increase. . Proposed method has $8 \%$ overshoot, it does not have rise time and the steady sate error in 30 second is about zero. In certain unstructured uncertainty, proposed method has good stability and robustness. Proposed controller has fantastic time response in presence of uncertainty. 


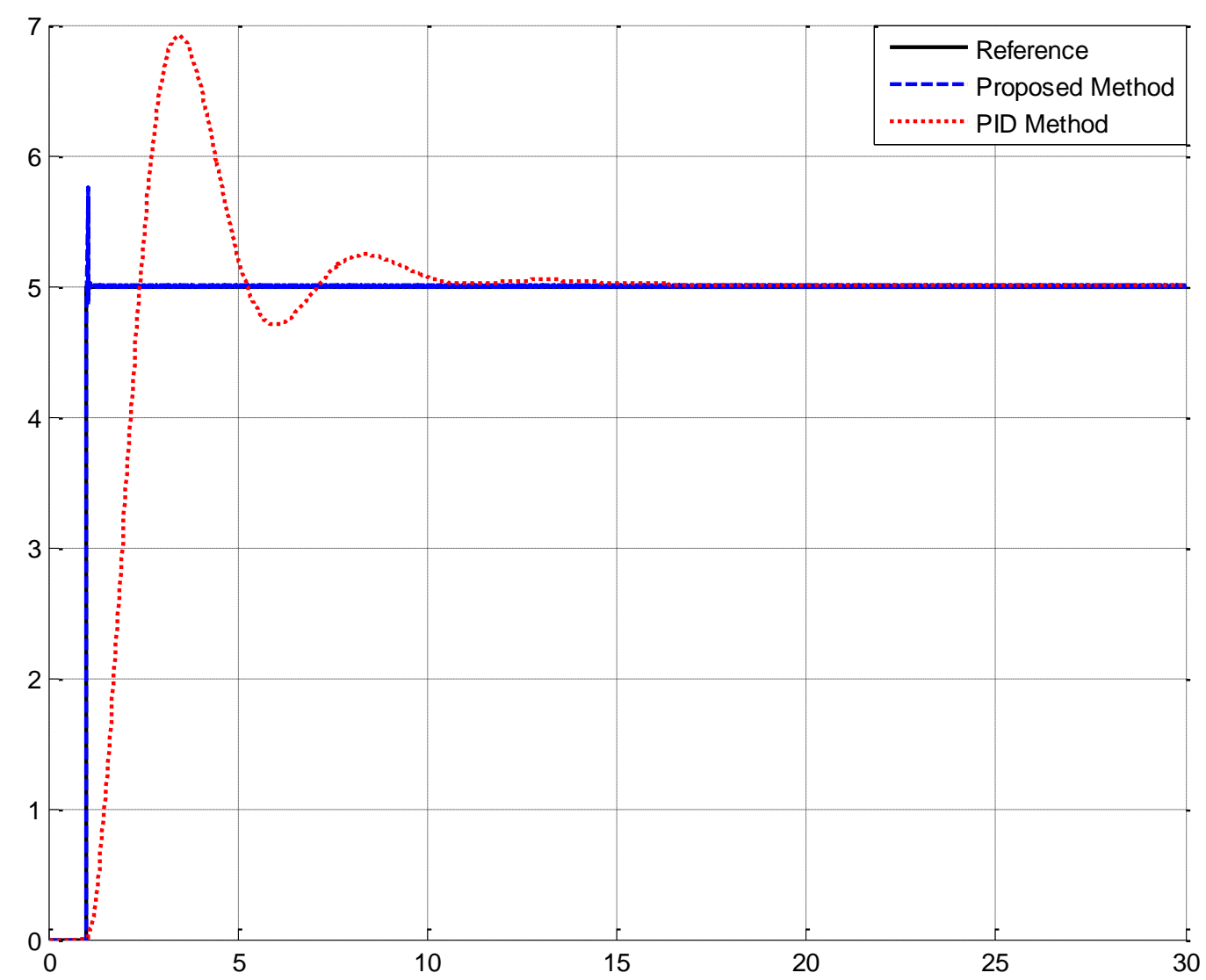

Figure 15. Disturbance Rejections for PID Controller and Proposed Method

Steady state error: Figure 16 shows the steady state error for PID controller and proposed method in presence of uncertainty. Regarding to the following Figure, PID controller has fluctuations in uncertain condition. Proposed method has an excellent result in certain and uncertain condition. 


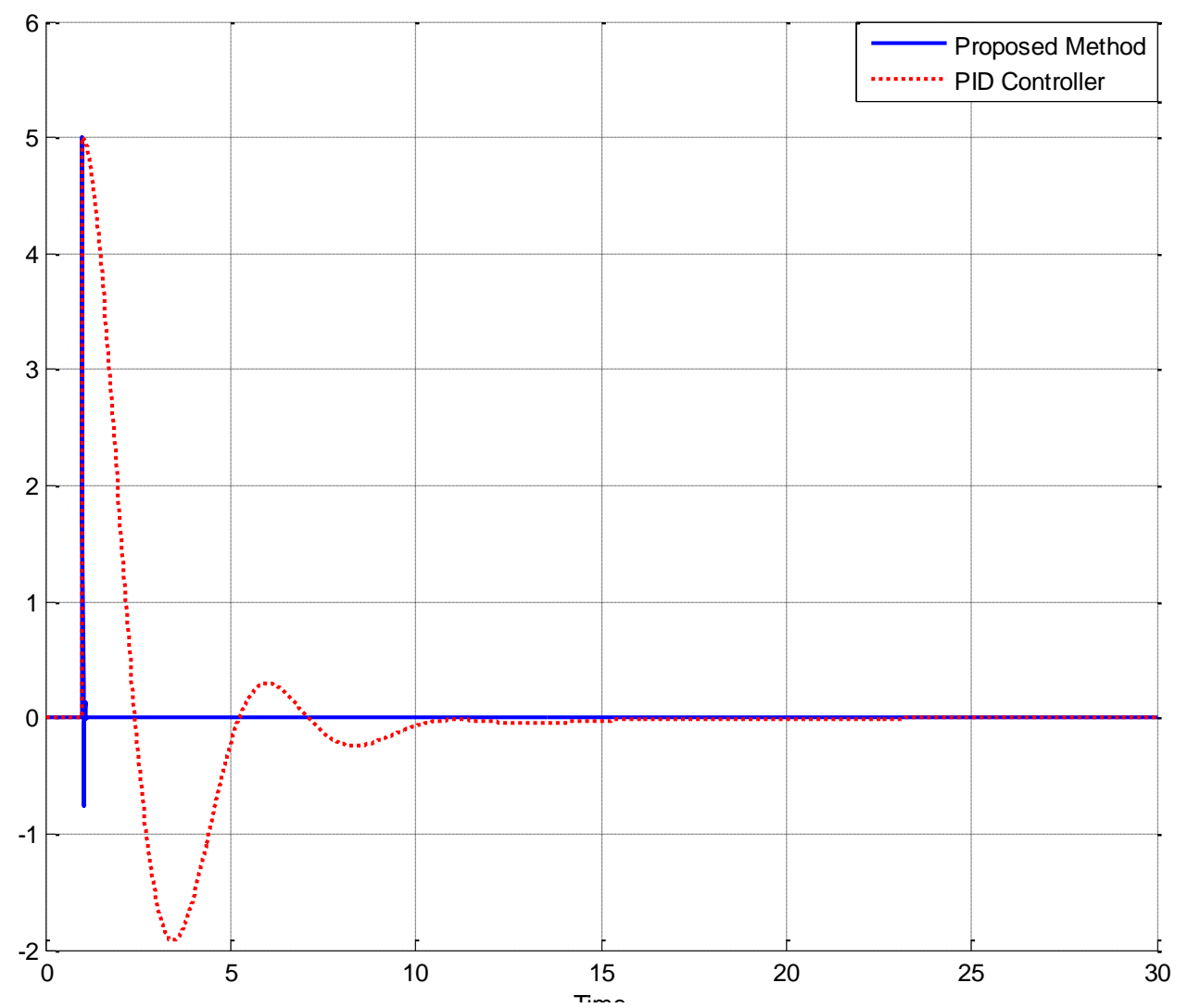

Figure 16. Steady State Errors for PID Controller and Proposed Method

\section{Conclusion}

In this research, design stable controller to control of three degrees of freedom dental joint is studied. First PID controller is candidate to this research. PID controller has 5\% overshoot and rate of signal change is 0.82 second in certain condition. PID controller has been acceptable performance in certain condition. PID controller was tested in unstructured uncertainty, there was a $40 \%$ overshoot and the rate of signal change was 1.3 second. Regarding to Figures 14 and 15 and with compare these two graphs, PID controller has stability and robust problems in presence of uncertainty. Filter PID fuzzy controller is the second candidate to control of this nonlinear and couple system. This controller has stable and robust. Proposed method has not any overshoot and the rate of signal change is about zero second in certain condition. Regarding to Figure 14 this method has fantastic response in certain condition. Proposed method has been tested in uncertain unstructured, it has $8 \%$ dip overshoot but the rate of signal change was about zero as well certain condition. Regarding to Figure 16 the rate of error change in proposed method was fantastic. Regarding to these graphs proposed method has acceptable performance to control of three degrees of freedom system. The PID like fuzzy controller with minimum rule base has been used in this research. Regarding to this research proposed method is stable and robust intelligent controller with minimum rule base. 


\section{Acknowledgment}

The authors would like to thank the anonymous reviewers for their careful reading of this paper and for their helpful comments. This work was supported by the Iranian Institute of Advance Science and Technology Program of Iran under grant no. 2013Persian Gulf-2A.

Iranian center of Advance Science and Technology (IRAN SSP) is one of the independent research centers specializing in research and training across of Control and Automation, Electrical and Electronic Engineering, and Mechatronics \& Robotics in Iran. At IRAN SSP research center, we are united and energized by one mission to discover and develop innovative engineering methodology that solve the most important challenges in field of advance science and technology. The IRAN SSP Center is instead to fill a long standing void in applied engineering by linking the training a development function one side and policy research on the other. This center divided into two main units:

- Education unit

- Research and Development unit

\section{References}

[1] Vachtsevanos, G. I., Davey, K. and Lee, K. M., "Development of a Novel Intelligent Robotic Manipulator," IEEE Control System Magazine, 1987, pp.9-15.

[2] Davey, K., Vachtsevanos, G. I., and Powers, R., "An analysis of Fields and Torques in Spherical Induction Motors," lEE Transactions on Magnetics, Vol. MAG-23, 1987, pp. 273-282.

[3] Foggia, A., Oliver, E., Chappuis, F., "New Three Degrees of Freedom Electromagnetic Actuator," Conference Record -lAS Annual Meeting, Vol. 35, New York, 1988.

[4] Lee, K. M., Vachtsevanos, G. and Kwan, C-K., "Development of a Spherical Wrist Stepper Motor," Proceedings of the 1988 IEEE Intemational Conference on Robotics and Automation, Philadelphia, PA. April 26-29.

[5] Lee, K. M., Pei. I., "Kinematic Analysis of a Three Degree-of-Freedom Spherical Wrist Actuator," The Fifth International Conference on Advanced Robotics, Italy, 1991.

[6] Wang, I., Jewel, G., Howe, D., "Modeling of a Novel Spherical Pennanent Magnet Actuator," Proceedings of IEEE International Conference on Robotics and Automation, Albuquerque, New Mexico, pp 1190-1195, 1997.

[7] Wang, I., Jewel, G., Howe, D., "Analysis, Design and Control of a Novel Spherical Pennanent Magnet Actuator," lEE Proceedings on Electrical Power Applications., vol. 154, no. 1, 1998.

[8] Chirikjian, G. S., and Stein, D., "Kinematic Design and Commutation of a Spherical Stepper Motor," IEEEIASME Transactions on Mechatronics, vol. 4, n 4, Piscataway, New Jersey, pp. 342-353, Dec. 1999.

[9] Kahlen, K., and De Doncker, R. W., "CW'l'ent Regulators for Multi-phase Pennanent Magnet Spherical Machines." Industry Applications Conference Record of the 2000 IEEE, vol. 3, 2000, pp. 2011-2016.

[10] Lee, K. M., Pei, I., and Gilboa, U., "On the Development of a Spherical Wrist Actuator," Proceedings of the 16th NSF Conference on Manufacturing Systems Research, Tempe AZ, January 8-12, 1990.

[11] Yang, C., Back, Y. S., "Design and Control of the 3-dcgn:es of freedom actuator by Controlling the Electromagnetic Force," IEEE Transactions on Magnetics, May, 1999, pp. 3607-3609

[12] Alireza Siahbazi, Ali Barzegar, Mahmood Vosoogh, Abdol Majid Mirshekaran, Samira Soltani,"Design Modified Sliding Mode Controller with Parallel Fuzzy Inference System Compensator to Control of Spherical Motor", IJISA, vol.6, no.3, pp.12-25, 2014. DOI: 10.5815/ijisa.2014.03.02

[13] Mojtaba Yaghoot, Farzin Piltan, Meysam Esmaeili, Mohammad Ali Tayebi, Mahsa Piltan,"Design Intelligent Robust Model-base Sliding Guidance Controller for Spherical Motor", IJMECS, vol.6, no.3, pp.61-72, 2014.DOI: 10.5815/ijmecs.2014.03.08

[14] Farzin Matin, Farzin Piltan, Hamid Cheraghi, Nasim Sobhani, Maryam Rahmani,"Design Intelligent PID like Fuzzy Sliding Mode Controller for Spherical Motor", IJIEEB, vol.6, no.2, pp.53-63, 2014. DOI: 10.5815/ijieeb.2014.02.07

[15] Ali Barzegar, Farzin Piltan, Mahmood Vosoogh, Abdol Majid Mirshekaran, Alireza Siahbazi,"Design Serial Intelligent Modified Feedback Linearization like Controller with Application to Spherical Motor", IJITCS, vol.6, no.5, pp.72-83, 2014. DOI: 10.5815/ijitcs.2014.05.10 
[16] Mohammad shamsodini, Farzin Piltan, Saman Rahbar, Ehsan Pooladi, Hossein Davarpanah,"Design New PID like Fuzzy CTC Controller: Applied to Spherical Motor ", IJMECS, vol.6, no.5, pp.60-68, 2014.DOI: 10.5815/ijmecs.2014.05.08

[17] Maryam Rahmani, Farzin Piltan, Farzin Matin, Hamid Cheraghi, Nasim Sobhani,"Design Intelligent System Compensator to Computed Torque Control of Spherical Motor", IJISA, vol.6, no.8, pp.87-96, 2014. DOI: 10.5815/ijisa.2014.08.10

[18] Mahsa Piltan, Farzin Piltan, Mojtaba Yaghoot, Saman Rahbar, Mohammad Ali Tayebi,"Design HighEfficiency Intelligent PID like Fuzzy Backstepping Controller for Three Dimension Motor", IJIEEB, vol.6, no.4, pp.52-62, 2014. DOI: 10.5815/ijieeb.2014.04.08

[19] Maryam Rahmani, Nasim Sobhani, Hamid Cheraghi, Farzin Piltan and Farzin Matin, “ Design Active Intelligent Multi Degrees of Freedom Joint Controller for Dental Automation", International Journal of Hybrid Information Technology, 8(10): 41-62, 2015. http://dx.doi.org/10.14257/ijhit.2015.8.10.05

[20] Mohammad Beheshti, Saman Rahbar, Hossein Davarpanah, Somayeh Jowkar and Farzin Piltan," Design Auxiliary Sliding Variable Sliding Mode Controller for Robot-Assisted Ophthalmic Surgery", International Journal of Bio-Science and Bio-Technology, 7(5): 187-202, 2015. http://dx.doi.org/10.14257/ijbsbt.2015.7.5.18. (Scopus, $S J R=0.24, Q 3)$

[21] Mohammad Reza Avazpour, Farzin Piltan, Mohammad Hadi Mazloom, Amirzubir Sahamijoo, Hootan Ghiasi and Nasri B. Sulaiman, " Research on First Order Delays System Automation", International Journal of Grid Distribution Computing, 8(4): 211-224, 2015. http://dx.doi.org/10.14257/ijgdc.2015.8.4.20.

[22] Ali Roshanzamir, Farzin Piltan, Arman Jahed, Saman Namvarchi, Iman Nazari, Nasri B. Sulaiman, “ Research on Nonlinear Automation for First Order Delays System”, International Journal of Hybrid Information Technology, 8(9): 313-328, 2015. http://dx.doi.org/10.14257/ijhit.2015.8.9.29

\section{Authors}

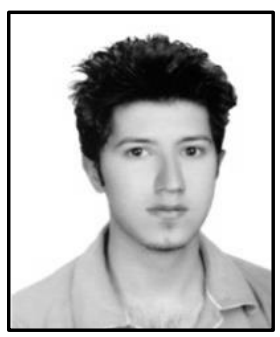

Farzin Matin has been working at "Design High Precision and Fast Dynamic Controller for Multi-Degrees of Freedom Actuator for Experimental Research and Education" project at Iranian Institute of Advance Science and Technology, Sanaat Kade Sabz Passargad Research Center (IRAN SSP) as "Student researcher " of a research team composed of 21 researchers since July. 2013 to July 2014.

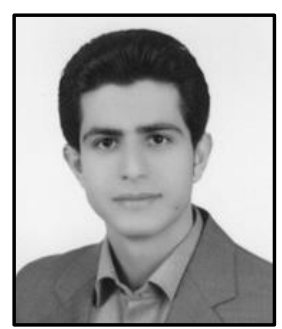

Hamid Cheraghi has been working at "Design High Precision and Fast Dynamic Controller for Multi-Degrees of Freedom Actuator for Experimental Research and Education" project at Iranian Institute of Advance Science and Technology, Sanaat Kade Sabz Passargad Research Center (IRAN SSP) as " Student researcher " of a research team composed of 21 researchers since July. 2013 to July 2014.

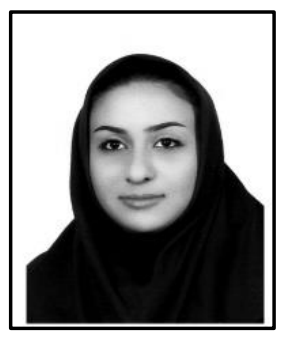

Nasim Sobhani has been working at "Design High Precision and Fast Dynamic Controller for Multi-Degrees of Freedom Actuator for Experimental Research and Education" project at Iranian Institute of Advance Science and Technology, Sanaat Kade Sabz Passargad Research Center (IRAN SSP) as "Student researcher " of a research team composed of 21 researchers since July. 2013 to July 2014. 


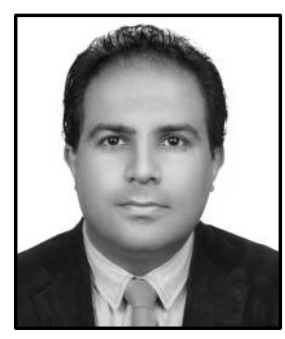

Farzin Piltan is an outstanding scientist in the field of Electronics and Control engineering with expertise in the areas of nonlinear systems, robotics, and microelectronic control. Mr. Piltan is an advanced degree holder in his field. Currently, Mr. Piltan is the Head of Mechatronics, Intelligent System, and Robotics Laboratory at the Iranian Institute of Advanced Science and Technology (IRAN SSP). Mr. Piltan led several high impact projects involving more than 150 researchers from countries around the world including Iran, Finland, Italy, Germany, South Korea, Australia, and the United States. Mr. Piltan has authored or co-authored more than 140 papers in academic journals, conference papers and book chapters. His papers have been cited at least 3900 times by independent and dependent researchers from around the world including Iran, Algeria, Pakistan, India, China, Malaysia, Egypt, Columbia, Canada, United Kingdom, Turkey, Taiwan, Japan, South Korea, Italy, France, Thailand, Brazil and more. Moreover, Mr. Piltan has peer-reviewed at least 23 manuscripts for respected international journals in his field. Mr. Piltan will also serve as a technical committee member of the upcoming EECSI 2015 Conference in Indonesia. Mr. Piltan has served as an editorial board member or journal reviewer of several international journals in his field as follows: International Journal of Control And Automation (IJCA), Australia, ISSN: 2005-4297, International Journal of Intelligent System and Applications (IJISA), Hong Kong, ISSN:2074-9058, IAES International Journal Of Robotics And Automation, Malaysia, ISSN:2089-4856, International Journal of Reconfigurable and Embedded Systems, Malaysia, ISSN:2089-4864.

Mr. Piltan has acquired a formidable repertoire of knowledge and skills and established himself as one of the leading young scientists in his field. Specifically, he has accrued expertise in the design and implementation of intelligent controls in nonlinear systems. Mr. Piltan has employed his remarkable expertise in these areas to make outstanding contributions as detailed follows:Nonlinear control for industrial robot manipulator (2010-IRAN SSP), Intelligent Tuning The Rate Of Fuel Ratio In Internal Combustion Engine (2011IRANSSP), Design High Precision and Fast Dynamic Controller For Multi-Degrees Of Freedom Actuator(2013-IRANSSP), Research on Full Digital Control for Nonlinear Systems (2011IRANSSP), Micro-Electronic Based Intelligent Nonlinear Controller (2015-IRANSSP), Active Robot Controller for Dental Automation (2015-IRANSSP), Design a Micro-Electronic Based Nonlinear Controller for First Order Delay System (2015IRANSSP).

The above original accomplishments clearly demonstrate that Mr. Piltan has performed original research and that he has gained a distinguished reputation as an outstanding scientist in the field of electronics and control engineering. Mr. Piltan has a tremendous and unique set of skills, knowledge and background for his current and future work. He possesses a rare combination of academic knowledge and practical skills that are highly valuable for his work. In 2011, he published 28 first author papers, which constitute about 
$30 \%$ of papers published by the Department of Electrical and Electronic Engineering at University Putra Malaysia. Additionally, his 28 papers represent about $6.25 \%$ and $4.13 \%$ of all control and system papers published in Malaysia and Iran, respectively, in 2011.

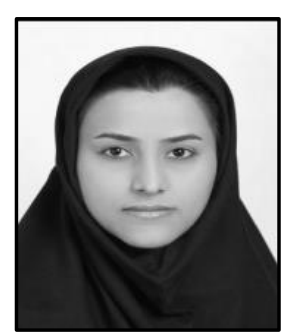

Maryam Rahmani has been working at "Design High Precision and Fast Dynamic Controller for Multi-Degrees of Freedom Actuator for Experimental Research and Education" project at Iranian Institute of Advance Science and Technology, Sanaat Kade Sabz Passargad Research Center (IRAN SSP) as "Student researcher " of a research team composed of 21 researchers since July. 2013 to July 2014. 